Qualitative study-other

\title{
Patients' perspectives on the impact of fasting while in hospital
}

$10.1136 /$ eb-2015-102166

\section{Cheryle Moss}

School of Nursing and Midwifery, Monash University, Clayton, Victoria, Australia

Correspondence to: Dr Cheryle Moss, School of Nursing and Midwifery, Faculty of Medicine, Nursing and Health Sciences, Monash University, Clayton Campus, Room C1.80, 10 Chancellors Walk (Formerly Building 13C), Clayton, VIC 3800, Australia; Cheryle.Moss@monash.edu

Commentary on: Carey SK, Conchin S, Bloomfield-Stone S. A qualitative study into the impact of fasting within a large tertiary hospital in Australia-the patients' perspective. J Clin Nurs 2015;24:1946-54.

\section{Implications for practice and research}

- This study, which involved qualitative analysis of 12 patients' experiences of fasting, needs replication internationally. Replication will reveal any consistencies in findings, and assist the development of international perspectives on this common and important clinical practice.

- Few studies investigate the experiences of patients in fasting. This study investigates two types of fasting that is, 'repeated' and 'prolonged'. Given the findings it will be important to investigate the experience of patients who undergo other types of fasting.

- There are implications for the person-centred care of patients who are fasting. Personal inquiry into the subjective and meaningful experiences of patients who are fasting while in their care, will help nurses to assess whether the care plan is effective, and what other aspects of care need development.

- The findings of this study, challenge nurses to identify best practice in care of patients who are fasting, and to examine the uptodateness of the fasting guidelines in use.

\section{Context}

While this qualitative study was undertaken in Sydney Australia in a large acute-care hospital, the preparation and management of patients who are fasting is central to nursing practice across the world. It is a common experience that patients may have repeated or prolonged fasting for procedures, or for management of their medical-surgical conditions. As the authors identify "there is minimal literature assessing the impact of fasting for patients". The aim of the study was to qualitatively explore the physical and emotional impact of fasting in hospital from the patients' perspectives.

\section{Methods}

After achieving ethical approval, adult in-patients $(n=12)$ who had been fasting for prolonged periods (or who had repeated fasting) were interviewed in this qualitative descriptive study. Semistructured interviews were designed to explore aspects of their experiences with food, their understandings and experiences of being nil-by-mouth, the extent to which staff were interested in their experiences of fasting, and their experiences of returning to eating. Interviews were recorded and transcribed, N-Vivo software was used to support data coding. Inductive thematic analysis was undertaken, and care was taken to achieve reasonable validity (credibility, transferability, dependability and neutrality).

\section{Findings}

The participants (aged 31-60 years) had a median fasting duration of 1.5 days (range 1-20). Most participants were interviewed postfasting. Participants aspects of fasting (thirst, dry mouth, changes in skin integrity). Some while fasting experienced physical relief from other symptoms. Other themes included: identified negative emotional impacts of fasting, the importance of food providing structure to the routine of an inpatient day, the difficulties of understanding nil-by-mouth jargon, fears about the consequences of the reintroduction of food. Frustration with the experience of unnecessary fasting was the experience of some.

\section{Commentary}

This important study adds possibilities for evidence-based practice when nurses work with patients who are fasting. Quality appraisal using the JBI Qari Appraisal Checklist ${ }^{1}$ for interpretive and critical research and the CASP Qualitative Research Checklist ${ }^{2}$ reveals that the research was of high quality. For example:

- The goal and rationale for the study are stated; qualitative description was a sound methodology for the inquiry.

- The research design and the methods of data collection were appropriate.

- There was congruity between the influence of the researcher, the methodology, research question, the research objectives, the methods of analysis.

- The researchers were careful in managing relationships between themselves and the participants, and ethical care was taken.

- The data analysis was rigorous.

- The themes were well illustrated by the use of participant voice.

Transferability in qualitative research is through insights gained from the results and the way that these invite clinicians to rethink, reflect on and modify their practices. After reading this paper nurses in practice will want to check the uptodateness of their current fasting regimes. This research stimulates motivation to achieve person-centredness in the care of patients who are fasting. The results highlight physical and emotional impacts of fasting, nurses could create nursing plans that seek to address these impacts. The routine use nil-by-mouth jargon by health professionals could be modified to make it more accessible for the lay community.

This research study highlights the need for more qualitative research to be undertaken with inpatients, so that new insights into their need for care are identified. There is a need for extension and replication of this research study. Are the seven themes identified sustained across different types of fasting regimes? Do the themes apply across different health services and in different cultures nationally and internationally? Answers to these and other related questions are needed. The research reported in the paper sets an important precedent, and generates the challenge for more knowledge and understanding about the experience of inpatients who are fasted during their care.

Competing interests None declared.

Provenance and peer review Commissioned; internally peer reviewed.

\section{References}

1. JBI QARI Critical Appraisal Checklist for Interpretive \& Critical Research. 2014. http://joannabriggs.org/assets/docs/jbc/operations/criticalAppraisalForms/JBC_Form_ CritAp_IntCrit.pdf (accessed 31 Oct 2015).

2. CASP Qualitative Research Checklist. 2015. http://www.casp-uk.net/\#! casp-tools-checklists/c18f8 (accessed 31 Oct 2015). 